\title{
Machine Learning Models for Government to Predict COVID-19 Outbreak
}

\author{
RAJAN GUPTA, Deen Dayal Upadhyaya College, University of Delhi, Delhi, India \\ GAURAV PANDEY and POONAM CHAUDHARY, The NorthCap University, Gurugram, India \\ SAIBAL K. PAL, Defence Research \& Development Organization, Delhi, India
}

The COVID-19 pandemic has become a major threat to the whole world. Analysis of this disease requires major attention by the government in all countries to take necessary steps in reducing the effect of this global pandemic. In this study, outbreak of this disease has been analysed and trained for Indian region till 10th May, 2020, and testing has been done for the number of cases for the next three weeks. Machine learning models such as SEIR model and Regression model have been used for predictions based on the data collected from the official portal of the Government of India in the time period of 30th January, 2020, to 10th May, 2020. The performance of the models was evaluated using RMSLE and achieved 1.52 for SEIR model and 1.75 for the regression model. The RMSLE error rate between SEIR model and Regression model was found to be 2.01. Also, the value of R0, which is the spread of the disease, was calculated to be 2.84 . Expected cases are predicted around $175 \mathrm{~K}-200 \mathrm{~K}$ in the three-week time period of test data, which is very close to the actual numbers. This study will help the government and doctors in preparing their plans for the future.

CCS Concepts: • Computing methodolongies $\rightarrow$ Machine learning algorithms; Mathematics of computing $\rightarrow$ Regression analysis;

Additional Key Words and Phrases: COVID-19, coronavirus, India, spread exposed infected recovered model, regression model, machine learning, predictions, forecasting

ACM Reference format:

Rajan Gupta, Gaurav Pandey, Poonam Chaudhary, and Saibal K. Pal. 2020. Machine Learning Models for Government to Predict COVID-19 Outbreak. Digit. Gov.: Res. Pract. 1, 4, Article 26 (August 2020), 6 pages.

https://doi.org/10.1145/3411761

\section{INTRODUCTION}

The 2019 novel coronavirus, better known as COVID-19, was reported in Wuhan, China, for the very first time on 31st December, 2019. According to Jiang et al. [1] the fatality rate for this virus has been estimated to be $4.5 \%$, but for the age group 70-79 this has gone up to $8.0 \%$, while for those $>80$, it has been noted to be $14.8 \%$. This has led to elderly persons above the age of 50 with underlying diseases such as diabetes, Parkinson's disease, and cardiovascular disease to be considered at the highest risk. Symptoms for this disease can take 2-14 days

Authors' addresses: R. Gupta, Deen Dayal Upadhyaya College, University of Delhi, Delhi, India; email: guptarajan2000@gmail.com; G. Pandey (corresponding author) and P. Chaudhary, The NorthCap University, Gurugram, India; emails: \{gauravpandey0801, poonam.potalia\}@gmail.com; S. K. Pal, Defence Research \& Development Organization, Delhi, India; email: skptech@yahoo.com.

Permission to make digital or hard copies of all or part of this work for personal or classroom use is granted without fee provided that copies are not made or distributed for profit or commercial advantage and that copies bear this notice and the full citation on the first page. Copyrights for components of this work owned by others than ACM must be honored. Abstracting with credit is permitted. To copy otherwise, or republish, to post on servers or to redistribute to lists, requires prior specific permission and/or a fee. Request permissions from permissions@acm.org.

(c) 2020 Association for Computing Machinery.

2639-0175/2020/08-ART26 \$15.00

https://doi.org/10.1145/3411761

Digital Government: Research and Practice, Vol. 1, No. 4, Article 26. Publication date: August 2020. 


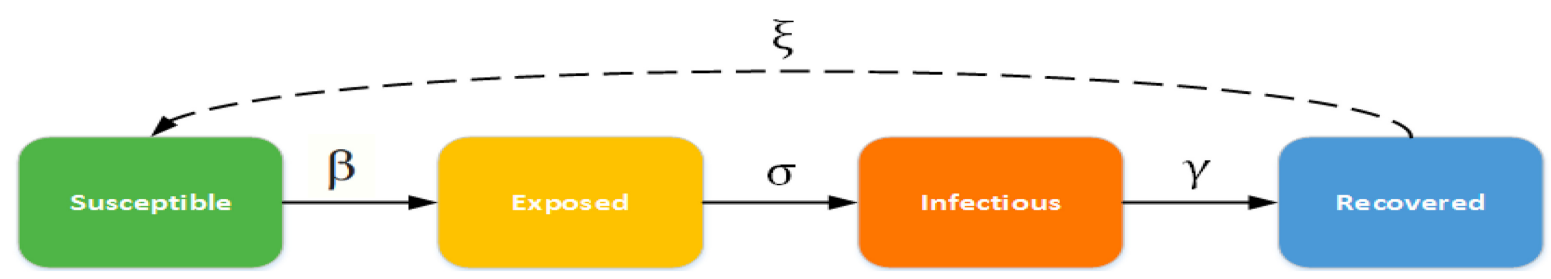

Fig. 1. Illustration of SEIR model and its four components.

to appear and can range from fever, cough, shortness of breath to pneumonia, kidney failure, and even death. The transmission is person-to-person via respiratory droplets among close contact, with the average number of people infected by a patient being 1.5-3.5, but the virus is not considered airborne [2]. An effective and well-tested vaccine against CoVID-19 has not been invented, and hence a key part in managing this pandemic is to decrease the epidemic peak, also known as flattening the epidemic curve. The role of data scientists and data mining researchers is to integrate the related data and technology to better understand the virus and its characteristics, which can help in making the right decisions and making concrete plans of action. It will lead to a bigger picture in taking aggressive measures in developing infrastructure, facilities, vaccines, and restraining similar epidemics in the future. The objectives of the current study are to use machine learning models to find the rate of spread of the disease in India using SEIR (Susceptible, Exposed, Infectious, Recovered) model and predict the number of cases through Regression and SEIR modelling techniques. Time series data provided by the Government of India web portal (https://www.mohfw.gov.in/) and John Hopkins University, USA, have been used for the empirical result analysis. The time period of training data is from 30/01/2020 to $10 / 05 / 2020$, and the test data from the time period 11/05/2020 to 31/05/2020. The data include confirmed cases, death cases, and recovered cases of all countries. However, this article focuses only on India's data for analysis and prediction of COVID-19 confirmed patients. The fact that India covers approximately $17.7 \%$ of the world's population and to date the effect of COVID-19 cases per million is less than 1 is the motivation behind this research.

\section{MACHINE LEARNING MODELS}

The two prominently used machine learning models are SEIR [3] and Regression model [4] for the disease infection forecasting around the world. The SEIR model has mainly four components, viz., Susceptible $(\boldsymbol{S})$, Exposed $(\boldsymbol{E})$, Infected $(\boldsymbol{I})$, and Recovered $(\boldsymbol{R})$, as shown in Figure 1. $\boldsymbol{S}$ is the fraction of susceptible individuals (those able to contract the disease), $\boldsymbol{E}$ is the fraction of exposed individuals (those who have been infected but are not yet infectious), $\boldsymbol{I}$ is the fraction of infective individuals (those capable of transmitting the disease), and $\boldsymbol{R}$ is the fraction of recovered individuals (those who have become immune).

The most important part of this model is to calculate the $R_{0}$ value. The value of $R_{0}$ tells about the contagiousness of disease. It is the fundamental goal of epidemiologists studying a new case. In simple terms $\mathrm{R}_{0}$ determines an average of what number of people can be affected by a single infected person over a course of time. If the value of $R_{0}<1$, this signifies the spread is expected to stop. If the value of $R_{0}=1$, this signifies the spread is stable or endemic. If the value of $\mathrm{R}_{0}>, 1$ this signifies the spread is increasing in the absence of intervention.

Regression models are statistical sets of processes that are used to estimate or predict the target or dependent variable on the basis of dependent variables. The regression model has many variants, such as linear regression, ridge regression, stepwise regression, polynomial regression, and so on. This study has used linear regression and polynomial regression [5] for prediction of COVID-19 cases. Equation (1) shows the relationship between a dependent and independent variable in polynomial regression. In Equation (1), $\boldsymbol{x}$ is the independent variable and $\boldsymbol{\theta}_{0}$ is the bias; also, the intercept and $\boldsymbol{\theta}_{1}, \boldsymbol{\theta}_{2}, \ldots \ldots, \boldsymbol{\theta}_{\boldsymbol{n}}$ are the weight or partial coefficients assigned to the predictors and $\boldsymbol{n}$ is the degree of polynomial.

$$
Y=\theta_{0}+\theta_{1} x+\theta_{2} x^{2}+\theta_{3} x^{3}+\theta_{n} x^{n} .
$$

Digital Government: Research and Practice, Vol. 1, No. 4, Article 26. Publication date: August 2020. 

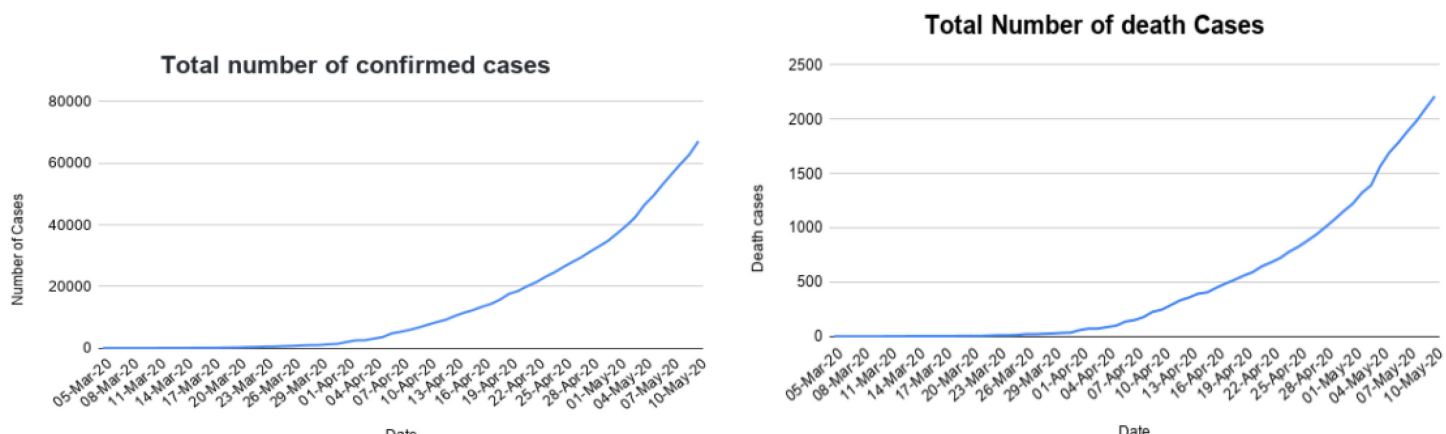

Fig. 2 (a) Shows the total number of confirmed cases due to COVID-19 in India till $10^{\text {th }}$ May. (b) Shows the total number of death cases in India till $10^{\text {th }}$ May.
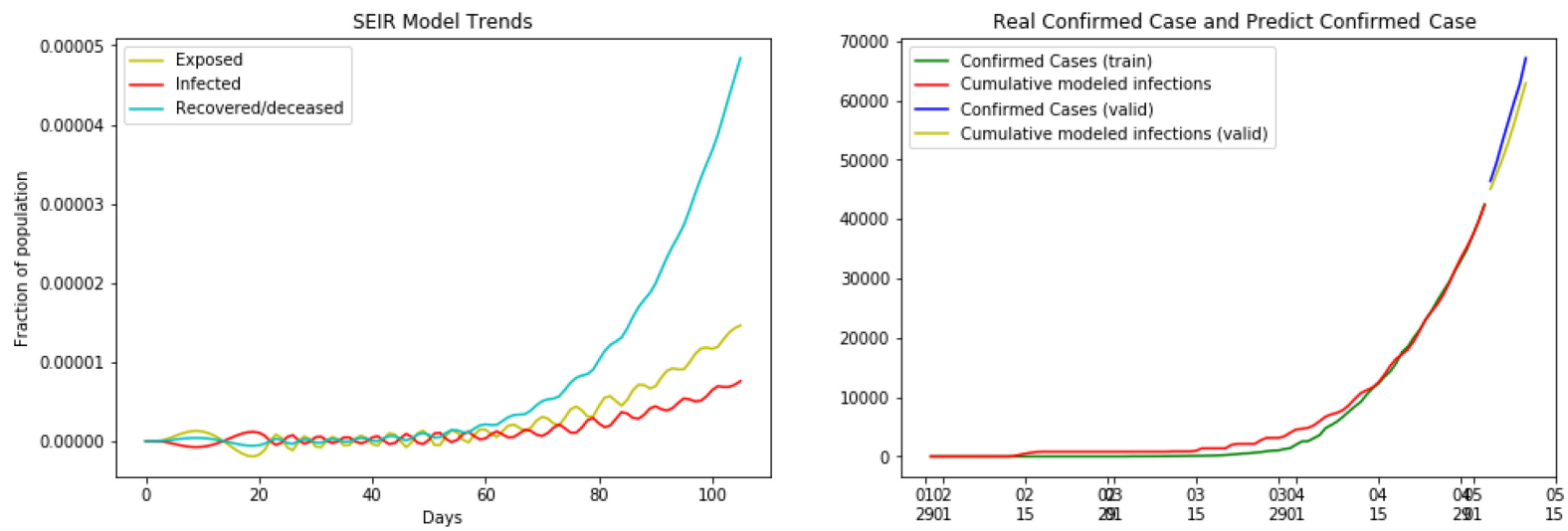

Fig. 3. Analysis using SEIR model. Left figure shows trend in SEIR model on India data, where yellow line shows the trend growth in exposed population, red line shows trend growth in infected population, cyan line shows trend growth in recovered or deceased population. Right figure shows the analysis by the SEIR model, where green line shows confirmed cases from training data, red line shows cumulative modelled infections, and blue line shows confirmed cases from validation data, yellow line shows cumulative modelled infections from evaluation set.

\section{ANALYSIS}

In India, the first case of COVID-19 was reported on 30th January, 2020. During the month of February, the number of cases reported was three and remained constant during the entire month. The major rise in the spread of the disease started in the month of March 2020. Figures 2 and 3 show the change in confirmed cases and death cases from 22nd January, 2020, to 10th May, 2020. Data from March show a significant change in spread of the disease.

In the current analysis, we have used data till 5th May, 2020, as our training data and data from 5th May, 2020-10th May, 2020, as the test/evaluation data. Before applying the prediction model, we analysed the time series training data to check whether the model can fit the data or not. We considered the result of confirmed cases in the $\log 10$ base versus last 30 days of training data. The reason for using last 30 days of training data is because it shows major growth in confirmed cases.

Then, we trained our data with SEIR model and result of line of fitting. While applying SEIR, we took care of some interventions such as quarantine and lockdown announced by the Government of India during this period, so, we applied a decay function to lower the number of confirmed cases during prediction. We used hill decay 
Table 1. Prediction of Number of COVID-19 Patients in India for Three Weeks Using SEIR and Regression Models

\begin{tabular}{|l|c|c|c|}
\hline Date & SEIR Model & Regression Model & Actual Cases in India \\
\hline 11-May-20 & 69.300 & 70.073 & 70.769 \\
\hline 12-May-20 & 72.942 & 74.052 & 74.331 \\
\hline 13-May-20 & 77.050 & 78.202 & 78.057 \\
\hline 14-May-20 & 81.460 & 82.530 & 82.048 \\
\hline 15-May-20 & 85.819 & 87.039 & 85.856 \\
\hline 16-May-20 & 89.763 & 91.735 & 90.650 \\
\hline 17-May-20 & 93.807 & 101.710 & 95.699 \\
\hline 18-May-20 & 98.325 & 107.000 & 100.327 \\
\hline 19-May-20 & 103.405 & 112.499 & 106.481 \\
\hline 20-May-20 & 108.845 & 118.214 & 112.201 \\
\hline 21-May-20 & 114.172 & 124.149 & 124.760 \\
\hline 22-May-20 & 119.051 & 130.312 & 131.425 \\
\hline 23-May-20 & 124.113 & 136.706 & 138.536 \\
\hline 24-May-20 & 129.670 & 143.340 & 144.950 \\
\hline 25-May-20 & 135.751 & 150.219 & 150.857 \\
\hline 26-May-20 & 142.106 & 164.737 & 158.103 \\
\hline 27-May-20 & 148.225 & 172.389 & 165.357 \\
\hline 28-May-20 & 154.190 & 180.311 & 173.495 \\
\hline 29-May-20 & 160.462 & 188.511 & 181.859 \\
\hline 30-May-20 & 167.185 & 196.994 & 190.648 \\
\hline 31-May-20 & 174.293 & 205.768 & \\
\hline
\end{tabular}

in our model, which is a half decay, and its formula is given in Equation (2), where L is a description of the rate of decay, $\mathrm{T}$ is time, and $\mathrm{k}$ is a shape parameter (no dimension). Half decay functions never reach zero and have half their original efficacies at time L.

$$
\text { hill decay function }=1 /\left(1+(T / L)^{k}\right) \text {. }
$$

For India region, from the training data the value of beta was computed equal to 0.6206 and value of gamma equal to 0.5106. Now using mathematical equations for SEIR model, we calculated the value of spread of disease or $\mathrm{R}_{\mathrm{o}}$ as equal to 2.84. Since we know COVID-19 is contagious and rate of transferring the disease from an infected person to susceptible person is 2.84, we need to predict the amount at which the disease can grow. Table 1 shows the prediction result using two models. In both the models, we have used 62 days' training data, as there was no significant trend in India before March 2020. To check the performance of the models used in this study, we used root mean squared log error (RMSLE), and the value of RMSLE for the SEIR model was found to be 1.52 , and 1.75 for the Regression model. The RMSLE error rate between SEIR model and Regression model was found to be 2.01. The value of RMSLE closer to zero is considered better. In this case, SEIR model has value of 1.52, which means the predicted value by SEIR and the actual values has a difference of 101.52 ( 34). Similarly for Regression model, the difference between predicted values and actual values is of $101.75(\sim 57)$.

\section{DISCUSSION \& CONCLUSION}

The current trend shows that there will be a linear trend continued in the next few days, as the control mechanisms taken by the Government of India are fairly strict and working well for the time being. The predicted and

Digital Government: Research and Practice, Vol. 1, No. 4, Article 26. Publication date: August 2020. 
actual cases are very close to each other. Also, with linear trends, the patients getting recovered can be managed easily and death rate can be controlled as well. The findings of the current study may explode exponentially, as shown by Gupta and Pal [6], if stringent control measures are not taken by the government. Hospital provisions and medical facility enhancement work should be continued at a very rapid pace to prepare the country for exponential growth. However, with current interventions and preparations, the Government of India is looking forward to flattening the curve.

During the prediction of the confirmed cases as shown in Table 1, there were few challenges associated with the data. The data were not stationary and showed an exponential growth after 40 days from 22nd January, 2020. Overfitting remains a major problem with disease spread time series data. In this model, we have addressed the overfitting problem using decay-based intervention. Another problem faced in this study was shortage of training data. Data for 62 days were used for training purpose and 5 days' data for validation, based on which the number of confirmed cases for the next 21 days was predicted. The training data are very less for any machine learning to train itself. Also, rapid changes in number of infected cases occurred in mid-March. The SEIR model shows an advantage, as it does not grow exponentially with time but also uses some intervention methods with time. For intervention, a hill decay model was used. In the case of a Regression model, features like the recovery rate, a decrease in growth rate can be used for decay. The growth of the regression line still remains a problem. For a Regression model, we always need to train the model after some time with the change in trend in the data. The SEIR model also uses some assumptions like the number of people in susceptible class, for which we have taken $70 \%$ of the population as susceptible class. In this study, we have only predicted the number of confirmed cases. To predict the number of death cases, we faced many problems of data stationarity. Also, with limited data, the model was not able to predict the number of death cases properly. We have used only time series data for confirmed cases and death cases in this study.

In this study, two machine learning models SEIR and Regression were used to analyse and predict the change in spread of COVID-19 disease. With the help of the SEIR model, the value of $\mathrm{R}_{0}$ was computed to be 2.84 . Also, we predicted the number of confirmed cases of COVID-19 for the next 21 days starting from 11th May, 202031st May, 2020, which was very close to the actual number of cases that happened in India. During performance evaluation, our model computed the value of RMSLE for the SEIR model to be $\mathbf{1 . 5 2}$ and $\mathbf{1 . 7 5}$ for the Regression model. Also, the value of spread of disease of $\mathrm{R}_{0}$ was found to be 2.84 .

The result obtained from this study is taken from training data up to 10th May, 2020. Further, looking at the trend, there is definitely going to be an increase in the number of cases. Doctors, health workers, and people involved in providing essential services have to be protected in accordance with prescribed medical norms. Community spreading in the future due to carelessness of individuals as well as groups can exponentially increase the number of cases. The peak is yet to come, hence the Government of India has to be extra vigilant and enforce strict measures. In addition, provision of medical facilities across the country has to be aggressively enhanced. In the future, an automated algorithm can be developed to fetch data in regular intervals and automatically predict the number of cases for weekly and bi-weekly data. In this way, government and hospital facilities can also maintain a check on the supply and medical assistance/isolation required for new patients. This study can be used by data science professionals from other regions for comparative analysis of various machine learning models on the Indian dataset. This study can also be used by administrators and health workers to assess the situation in the future.

\section{REFERENCES}

[1] F. Jiang, L. Deng, L. Zhang, Y. Cai, C. W. Cheung, and Z. Xia. 2020. Review of the clinical characteristics of coronavirus disease 2019 (COVID-19). F. Gen. Intern. Med. 35 (2020), 1545-1549.

[2] Z. Wu and J. M. McGoogan. 2020. Characteristics of and important lessons from the coronavirus disease 2019 (COVID-19) outbreak in China: Summary of a report of 72,314 cases from the Chinese Center for Disease Control and Prevention. FAMA 323, 13 (2020), 1239-1242. DOI : 10.1001/jama.2020.2648

[3] M. Y. Li and J. S. Muldowney. 1995. Global stability for the SEIR model in epidemiology. Math. Biosci. 125, 2 (1995), 155-164. 
[4] S. Yang, P. Cao, P. Du, Z. Wu, Z. Zhuang, L. Yang, X. Yu, Q. Zhou, X. Feng, X. Wang, W. Li, E. Liu, J. Chen, Y. Chen, and D. He. 2020. Early estimation of the case fatality rate of COVID-19 in mainland China: A data-driven analysis. Ann. Trans. Med. 8, 4 (Feb. 2020).

[5] N. S. Punn, S. K. Sonbhadra, and S. Agarwal. 2020. COVID-19 epidemic analysis using machine learning and deep learning algorithms. Retrieved from https://www.medrxiv.org/content/10.1101/2020.04.08.20057679v2.

[6] Rajan Gupta and Saibal K. Pal. 2020. Trend analysis and forecasting of COVID-19 outbreak in India. Retrieved from https://www.medrxiv. org/content/10.1101/2020.03.26.20044511v1.

Received June 2020; accepted June 2020

Digital Government: Research and Practice, Vol. 1, No. 4, Article 26. Publication date: August 2020. 